\title{
Pediatric Osteosarcoma: Connecting Present and Future Approaches to Diagnosis and Treatment
}

\author{
Konstantinos Kapetanos ${ }^{1 *}$, Dimitrios Asimakopoulos ${ }^{1}$, Georgia Savvides ${ }^{2}$ and Anna Vavlitou ${ }^{3}$ \\ ${ }^{1}$ University of Cambridge, School of Clinical Medicine, UK \\ ${ }^{2}$ University of Manchester, School of Clinical Medicine, UK \\ ${ }^{3}$ Intensive Care Unit, Nicosia General Hospital, Cyprus
}

*Corresponding author: Konstantinos Kapetanos, University of Cambridge, School of Clinical Medicine, UK

\section{ARTICLE INFO}

Received: 幽 November 30, 2020

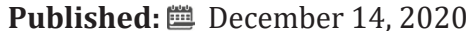

\section{Citation: Konstantinos Kapetanos, Dimitrios Asimakopoulos, Georgia Savvides, Anna Vavlitou. Pediatric Osteosarcoma: Connecting Present and Future Approaches to Diagnosis and Treatment. Biomed J Sci \& Tech Res 32(3)- 2020. BJSTR. MS.ID.005268.}

Keywords: Pediatric Osteosarcoma; Radiotherapeutic Techniques; Metastasis; MicroRNA; Methotrexate; Immunological Treatments

\begin{abstract}
Pediatric osteosarcoma is a highly aggressive type of cancer, affecting children of all ages. Early diagnosis is key to ensure better outcomes, as pre-metastatic lesions are easier to manage. For this reason, novel screening techniques, such as miRNA biomarkers, have been developed to complement the standard radiological approach to diagnosis. Furthermore, the standard of care in chemotherapeutic treatment is constantly revised, and new drugs are designed to minimize the toxic effects of the treatment whilst maintaining, or even improving, its efficacy. Immunotherapy and radiotherapy are two more disciplines which have demonstrated great promise in their capacity to exert strong anti-tumor properties in a clinical setting. Immunotherapeutic drugs like Mifamurtide, and radiological treatments such as proton beam therapy are starting to form an essential part of the golden standard of care. Lastly, innovations in orthopedic oncology provide surgical techniques with the potential to improve surgical outcomes whilst preserving as much of the patient's healthy tissue as possible. This review appreciates that there is a great promise held in the future of osteosarcoma treatment, and highlights where attention should be paid in the future.
\end{abstract}

Abbreviations: OS: Pediatric Osteosarcoma; PDGFR: Platelet Derived Growth Factor Receptor; VEGFR: Vascular Endothelial Growth Factor Receptor; MHC: Multiple Histocompatibility Complex; LSS: Limb-Salvage Surgery; MSTS: Musculoskeletal Tumor Society

\section{Introduction}

Pediatric Osteosarcoma (OS) is a highly aggressive type of cancer that represents $2.7 \%$ of all pediatric cancers [1]. It usually manifests in the first two decades of life, and it poses a particular challenge to treat, due to its highly unstable genetic and epigenetic nature [2]. Over the years, the management of this disease has evolved greatly, via an evolution that paralleled the changing landscape of science and technology during the late twentieth century. This has come through improvements in; (i) means of early diagnosis, (ii) medical and immunological treatments, (iii) surgical technologies and (iv) radiotherapeutic techniques. However, over the past few years, the field has stagnated and several limitations of the current approach to treatment have been revealed. Therefore, it is important to understand the current state of the art regarding the management of OS, acknowledge its limitations and appreciate all research attempts in the field that might yield a useful addition to the standard of care in the near future.

\section{Diagnosis}

\section{The Importance of an Early Diagnosis}

OS is an aggressive cancer with prognostic outcomes depending on whether detection occurs prior to metastasis. Specifically, it is shown that misdiagnosis and consequent delay in treatment are associated with inferior survival [3], as there is an approximately $60-70 \%$ survival rate in patients without metastasis, contrasting a survival rate of $20-30 \%$ in patients with metastasis [4]. Furthermore, $80-90 \%$ of diagnosed cases of osteosarcoma are high grade, further supporting the aggressive nature of the disease [5]. With metastasis changing prognostic outcomes to this extent 
it is important to catch the cancer early to improve survival rates among the patient cohort. There are several factors that make this a tough feat to accomplish: (1) the vague clinical presentation of osteosarcoma, (2) the lack of knowledge of the disease by primary care and (3) the lack of multidisciplinary action, which can lead to misdiagnosis [6,7]. Therefore, it is important to understand the most efficient technique that will permit an accurate diagnosis.

\section{Means of Reaching A Diagnosis}

The diagnosis of Osteosarcoma using plain radiograph is the main method of diagnosis and can easily picture the stereotypical radiographic characteristics of the disease: dense sclerosis of the metaphysis (present in nearly all patients with osteosarcoma), soft tissue extension (75\%), Sunburst radiating calcification (50\%), osteosclerotic lesions (45\%), lytic lesions (30\%) [4,8]. Following suspicion raised by radiological findings, biopsy is performed to confirm the diagnosis. However, the use of X-ray is not economically sustainable, or necessary, for every child presenting with the most common symptom of osteosarcoma, joint pain, which has an array of differential diagnoses [9]. Furthermore, there is a strong association between low SES (Socio-Economic Status) and subscription to public insurance programs, with delayed times of diagnosis [10]. This highlights the need for developing more sustainable approaches to diagnosis that can be more readily available in a primary care setting. A method that may assist in early diagnosis of the disease is the upcoming use of microRNA in diagnosis. This is a type of endogenous nonprotein-encoding RNA which circulates in the serum. There is a differential expression of specific miRNA clusters in OS patients. There is a correlation of these miRNA clusters with stage and grade of the tumor as well as if the patient has lung metastasis [11]. The use of these miRNA clusters as a means of diagnosis reports a 79-80\% sensitivity and $77-89 \%$ specificity in detecting osteosarcoma patients from controls $[12,13]$. This may be an economically friendly, easy and efficient method for the detection of early osteosarcoma and may result in better patient outcomes in the future, preventing the risk that comes with not giving an X-ray.

\section{Medical Treatment}

\section{Chemotherapy}

Chemotherapy lies at the centre of all strategies implemented in the medical management of Pediatric Osteosarcoma (OS). The idea of using chemotherapeutic agents to treat OS was introduced by Jaffe in the 1970s [14], who highlighted the potential of methotrexate in bone-tumor suppression. The idea, however, remained controversial until a hallmark study by Link, a decade later, revealing the capacity of multi-agent chemotherapy to extent 5-year survival rates of OS patients [15]. This introduction of multi-agent chemotherapy in the management of OS pushed survival rates from $<15 \%$ to over $65 \%$, and until now, acts as a major checkpoint in the advancement of the field. Many of the agents included in the study by Link et al., are still used today as the mainline treatment regimen. Specifically, most treatment protocols around North America and Europe use the MAP regimen (Methotrexate, Adriamycin and Cisplatin) as the primary adjuvant chemotherapeutic cocktail, before and after surgical tumor resection [16-18]. Since then, little progress has been observed in the field of chemotherapeutic treatment of OS [19]. "EURAMOS" marks a recent multi-centre international attempt to investigate the efficacy of novel treatments and explore the introduction of new agents like Isosfamide and Etoposide in the mainstream treatment regimen (i.e. MAPIE vs MAP). The study concluded that the introduction of two more chemotherapeutic agents markedly increased the level of toxicity inflicted by the treatment regimen, without any significant improvement in survival outcomes [20]. Gemcitabine is another drug that has recently made its way into clinical trials for OS, and so far, has shown promising results [21], but its true impact remains to be appreciated. The reasons why there has been little advancement in the field primarily relate to the highly aggressive and genetically unstable nature of OS, as well as the highly toxic features of chemotherapy. Therefore, it can be argued that any improvement in the survival rates of OS patients ought to come from other modes of treatment.

\section{Future Treatments}

The potential of traditional chemotherapeutic approaches to the treatment of OS is limited by their toxic adverse effects and the resistance to therapy exhibited by cancer cells. Therefore, the design of novel therapeutic agents takes an alternative approach and attempts to hijack specific pathways in order to alter the behaviour of tumor parenchyma and stroma [22]. Specifically, this can be done by the application of drugs that interfere with cancer cell metabolism and halt proliferation, by inhibiting the function of the mammalian target of rapamycin (mTOR). For example, both sirolimus and everolimus have exhibited a potential of limiting tumor growth in OS cells during preclinical studies [23-25]. Furthermore, there is potential for therapies that work on the tumor microenvironment to inhibit angiogenesis and ECM remodeling. For example, Pazopanib, an inhibitor of Platelet Derived Growth Factor Receptor (PDGFR), Vascular Endothelial Growth Factor Receptor (VEGFR) and c-kit, and Sorafenib, an inhibitor of VEGFR-2 and PDGFR-B, have both shown promising results in small, singlecentre clinical trials [26,27]. Moreover, bisphosphonates are a recently introduced class of drugs that can have a multifocal effect, by arresting proliferation, promoting apoptosis and inhibiting angiogenesis in cancer tissue. Zoledronate is an example of a bisphosphonate drug that has demonstrated promising results, and is now included in large clinical trials alongside combination chemotherapy [28]. Lastly, genetic studies trying to understand the genetic and epigenetic basis of osteosarcoma have yielded great results that have the potential of being used for the development of even more sophisticated treatments [2] For example, Caronia et al. 
have identified a group of Single Nucleotide Polymorphisms (SNPs) that are predominantly expressed in OS and which are theorised to promote resistance to chemotherapy [29] Appreciating the importance of the results stemming from genetic studies can pave the way for designing more effective, personalized treatments.

\section{Immunotherapy}

\section{Tumor Vaccines}

A treatment that has recently gained traction in the field of medical oncology is immunotherapy, a form of cancer therapy that promotes the recruitment of the patient's own immune system to fight off the invading cancer. Tumor vaccines are one of the strategies equipped when fighting off cancers such as osteosarcoma and involve the introduction of an immunogenic substance that elicits an inflammatory response and activates the immune system against a specific Tumor-Associated Antigen (TAA). Perhaps the most successful attempt towards the development of a tumor vaccine against OS is MTP (Muramyl Tripeptide) and its associated molecule, L-MTP-PE (Liposome-encapsulated MTP phosphatidylethanolamine), currently used under the brand name "Mifamurtide". MTP is a molecule that resembles the outer surface of bacterial walls and its introduction to the patient's blood is enough to elicit an immune response defined by the presence of inflammatory cytokines (TNF $\alpha$, IL-6) and monocyte activation [30]. This response exhibits anti-tumor properties, and several clinical trials highlight its capacity to improve survival rates in OS patients, alongside its safety to be used in parallel with standard chemotherapy [31,32]. For these reasons, the European Medicine agency has approved its use as a mainline treatment. Similar attempts to create an effective tumor vaccine against OS have been taken, all centered around creating an agent that will elicit a similar cytokinetic response. For example, the use of a mixed bacterial vaccine alongside allogenic tumor tissue has exhibited antitumor properties in a clinical setting [33], attributed to its capacity to elicit a strong inflammatory cytokinetic profile of IL-6, TNF $\alpha$, IFN $\gamma$ and IL-1 $\beta$ [34]. Furthermore, direct injection of inflammatory cytokines IFN $\alpha$ and IL- 2 were seen to elicit antitumor responses and have demonstrated promising results in clinical trials $[35,36]$. However, few attempts have reached results as significant as the application of L-MTP-PE in a clinical setting, and which acts as the current golden standard against which any new immunotherapeutic approach to OS treatment will be measured.

\section{Cellular Immunotherapy}

Cellular immunotherapeutic techniques target the arousal of an adaptive (T-cell mediated) response against tumor tissue. To begin with, Adoptive T-cell therapy is a technique that relies on the artificial construction of a T-Cell Receptor (TCR), which will recognise and respond to specific TAAs. This was attempted by the introduction of the genes for TCR $\alpha$ and $\beta$ chains into a T-cell population, creating a TCR specific for the antigen NY-ESO-1. This elicited positive outcomes when used against NY-ESO-1+ synovial sarcomas [37]. An alternative approach is the construction of a chimeric T-cell receptor (CAR), which permits the Multiple Histocompatibility Complex (MHC-) independent Antigendependent T-cell activation. The application of CAR T-cell therapy in creating a response against a HER2 antigen in patients with recurrent/refractory sarcoma, demonstrated a capacity for halting disease progression in 4/17 patients [38]. However, there are several risks noted with this approach, such as the manifestation of a cytokine storm or the precipitation of an autoimmune response [39]. Moreover, an alternative approach to cellular immunotherapy is checkpoint inhibition, whereby the blockade of certain molecules that function as "immunological checkpoints" permits the activation of an immunological response against the cancer tissue. Namely these molecules are Cytotoxic T-Lymphocyte-Associated Protein 4 (CTLA4) and Programmed Cell Death Protein 1 (PD1). This technique is widely established as effective in multiple types of cancer, including; lung cancer, melanoma, renal cell carcinoma and ovarian cancer, and is used as part of their mainline treatment (Nobel prize in physiology or medicine 2018).

However, limited evidence supports the use of either ipilimumab (inhibitor of CTLA4) or nivolumab (inhibitory of PD-1) in patients with pediatric osteosarcoma. There is some evidence to suggest that PD-1 inhibition can promote the prevention or regression of lung metastases secondary to OS in mouse models, but no effect is observed on the primary cancer tissue [40]. Furthermore, combined PD-1 and CTLA-4 inhibition has elicited some positive effect on the overall survival of OS mouse models when compared to the use of one form of inhibition alone [41]. However, the true potential of checkpoint inhibition in primary sarcomas remains to be appreciated [39].

\section{Surgery \& Reconstruction}

\section{Tumor Resection}

Prior to the 1970s, surgical management of localised osteosarcoma (i.e. not metastasised to distal sites) almost exclusively involved ablation surgery; i.e. amputations, rotationplasties and disarticulations [42-44]. By 1990, amputations had become less frequent as Limb-Salvage Surgery (LSS) was introduced and displaying was demonstrating more promising results [45]. LSS is a two-step process that involves the removal of the tumor by safe margins, followed by surgical reconstruction of the affected bones. The introduction of LSS coincided with advancements in chemotherapy and CT imaging, and during that time the 5-year survival rates of osteosarcoma patients rose rapidly, from 10-15\% in the 1970 s [22] to $>50 \%$ in the 1980 s and $>90 \%$ by the early $21^{\text {st }}$ century [46]. Direct comparison of the two practices (amputation vs LSS) today, highlights marked improvements in survival rates, metastasis occurrence rates and functional outcomes of patients with limb osteosarcomas [47]. Specifically, os patients that 
undergo amputation surgery are less likely (OR 0.50-0.75) to reach the 5-year survival than patients undergoing LSS [48,49], and more likely to exhibit lower Musculoskeletal Tumor Society (MSTS) scores assessing functional outcomes [49]. For those reasons, LSS is currently the golden standard of surgical treatments, used in $>90 \%$ of cases, and provides a safe approach to treatment in $85-90 \%$ of those cases [50]. However, there is still some evidence suggesting that amputation might be the most appropriate approach when the tumor margins are not clear, as it can ensure a much lower risk of local recurrence [51]. It is therefore important to appreciate how each patient presentation is unique and the approach to their treatment should be tailored accordingly.

\section{Reconstruction}

Following tumor resection during LSS, there are several approaches that can be taken to restore the functional integrity of the affected limb. This largely depends on the functional deficits inflicted by the tumor excision and non-weight bearing bones, like the proximal fibula or the clavicle, generally don't require reconstruction [52]. In cases where the tumor resection results in the removal of a joint, the primary approach to reconstruction is joint replacement by an artificial implant (Figure 1). There are several advantages to this approach, including the simple surgical technique, the stability and flexibility (range of motion $60-110^{\circ}$ ), the high 5-year survival rates (87\%) and the great extent of functional recovery [53]. However, there are still some major limitations in joint replacement following LSS in pediatric OS patients. Firstly, there is a marked risk of infection, as high as $10 \%$, due to the requirement for osseointegration of the implant [43]. Furthermore, the exposure of the implant to repetitive impact forces can cause bushing wear, creating the need for multiple revision surgeries [43]. Lastly, the introduction of an artificial joint in an adolescent patient can impair the epiphyseal plate, creating the risk of difference in limb length at the end of puberty [54].

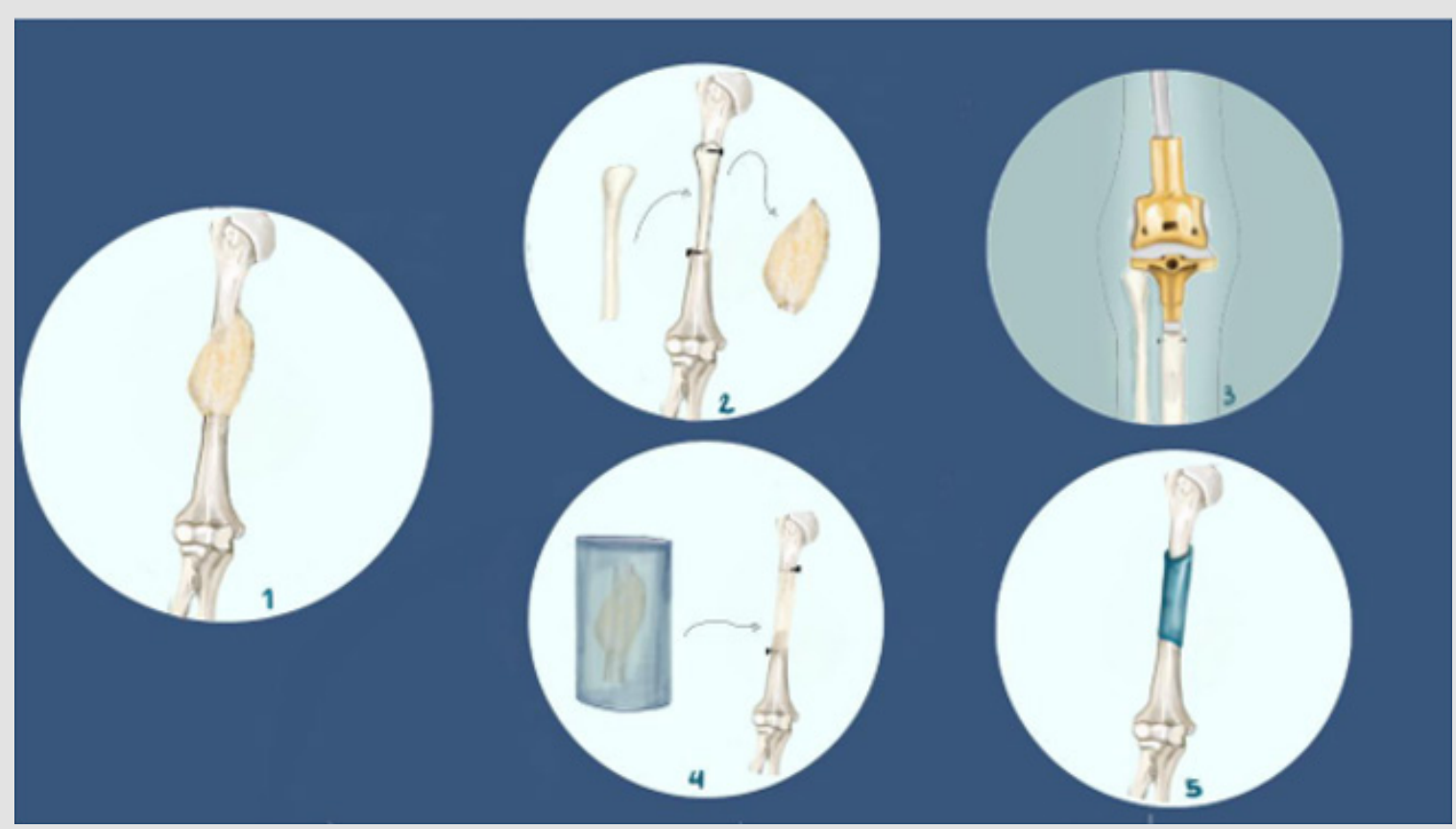

Figure 1: Schematic of strategies for reconstruction following LSS (Limb Salvage Surgery).

1. Osteosarcoma tumor on the humerus.

2. Free vascularized fibula graft applied in humerus reconstruction.

3. Joint replacement by mechanical implant (golden standard)

4. Tumor devitalized autograft prepared by either freezing. It should be noted that other devitalization techniques include bone pasteurization and irradiation, which are not shown in this figure.

5. Custom made implant applied in humerus reconstruction.

6. Parts 2,4,5 are strategies used in JPS (Joint Preservation Surgery), whereby the affected joint is preserved rather than replaced.

The above limitations highlight the need to explore further surgical options, including Joint-Preservation Surgery (JPS), which is introduced as an alternative to joint replacement surgery. The main concept of reconstruction in JPS is to replace the excised portion of the affected bone or joint with the patient's own tissue (autograft). There are several techniques via which an autograft can be created, each presenting with a different set of outcomes, namely; tumour-devitalised autografts, free vascularized fibular 
grafts and custom-made implants (Figure 1). Although variable, there is strong evidence to support the effectiveness of all four techniques in terms of survival and recurrence rates in pediatric osteosarcoma patients [54]. For example, in a trial using freezingmediated tumor-devitalization technique (Figures 1a \& 1b), excellent functional outcomes were reported in 15/18 patients that received frozen autografts, and the ability to walk without a mobility aid in 16/18 [55]. Furthermore, Kamal \& Rubiansyah report a higher survival rate in the case of extracorporeal irradiation (Figure 1c) of the affected joint, at 94.6 months, vs. 90.9 months for megaprosthetic implants. JPS also has the advantage of being able to better protect the epiphyseal plate in certain pediatric OS patients [56], and thereby avoid the risk of causing a limb length discrepancy. These data, although also showing that extracorporeal irradiation is associated with lower functional outcomes and higher recurrence rates, may set the ground for JPS becoming the mainstream surgery in the future, with the refinement of existing surgical technologies [54,57].

\section{Outcome}

The outcome of LSS surgery is multifactorial and complicated. Among the factors that have to be considered include sex (male to female OR = 1.21), age $(12-20 \geq$ vs. $<12 \mathrm{OR}=1.37)$, site (proximal vs. distal), grading and staging of the tumor, delay until referral and response to chemotherapy, and patient age [47,48,58]. A factor that significantly decreases patient survival is previous invasive surgery on the affected site, including due to fractures, and due to the increased likelihood of unclear surgical excision margins, and consequently, higher recurrence rates [48]. In particular, infections of the proximal tibia and the pelvis, which are more likely in immunocompromised patients and fractures, act as contraindications against surgery [43]. These data highlight that surgical management of OS is only a part of a multidisciplinary approach to the treatment and that there are several factors that will determine the outcome of a pediatric patient.

\section{Radiation Therapy}

\section{Radiotherapeutic Technologies}

Radiotherapy is one of the available options for the management of osteosarcoma, with both curative and palliative intent, depending on its use, as well as relatively unclear survival outcomes $[59,60]$. External beam radiotherapy is the standard form of radiotherapy, and it is specific to the administration of radiation from an external radiation source. There are several techniques via which external beam radiotherapy can be applied, each one using a variable form of radiation, 3D conformal, Intensity-Modulated Radiation Therapy (IMRT) and intensity-modulated proton therapy [61,62]. When applied to the management of OS, external beam radiotherapy seems to achieve higher survival rates when used to treat primary tumours (55\% 5-year survival rate-SR), when compared to locally recurrent $(15 \% \mathrm{SR})$ or metastatic $(0 \% \mathrm{SR})$ cases and its effects can potentiated by the addition of radionuclide agents, such as Samarium-153 [63,64]. Schwarz et al. further showed that there is a much greater local control rate of $48 \%$, when combining radiotherapy with surgery, as opposed to $22 \%$ in radiotherapy alone. This illustrates the need for a multidisciplinary approach to optimize survival rates [63]. Furthermore, Tinkle et al. reported 5 -year survival rates of $54.6 \%$ vs. $24.3 \%$, respectively, when comparing the use of radiotherapy at the stage of initial diagnosis vs. recurring illness [62]. Persistent tumours are reported to show shorter survival rates in other papers too [59].

With regards to proton-based radiotherapy, there is still limited evidence regarding its usefulness in the treatment of osteosarcoma. On the one hand, Ciernik et al. reported five-year disease-free survival and overall survival rates at $65 \%$ and $67 \%$, respectively. On the other hand, high rates of toxicity were also reported among the patient group (30.1\%), raising concerns regarding the overall costbenefit of this method [60]. Furthermore, an important limitation of radiotherapy is its association with the potential for infection, the rates of which are still questionable $[43,50,65]$.

\section{Conclusion}

The treatment of pediatric osteosarcoma has evolved greatly throughout the years. Advancements in technology have enabled the means of rapidly reaching an accurate diagnosis through $\mathrm{x}$-ray imaging. Then, a combination of chemotherapeutic, immunological, radiological and surgical approaches to treatment, have allowed a relatively safe management of the localised disease. However, there are marked limitations in all forms of treatment available to date, which is reflected in the poor outcomes of patients with metastatic disease. Therefore, focus should be turned onto novel areas of research, as they will hold the key for any future advancements to come. Currently, there is an endless stream of novel and innovative research, in labs and clinics alike, trying to advance the field further. Techniques such as bio-screening for miRNA markers appear able to complement radiographic investigations when trying to reach an early diagnosis. Novel pharmacological approaches, using small molecule-drugs, attempt to combat the progression of the disease with an unprecedented level of specificity. Specifically, bisphosphonate drugs appear to be a few steps away from being introduced in the standard regimen, as they exhibit high efficacy and low toxicity in late-stage clinical trials. Immunotherapeutic approaches are also presenting with promising results, highlighting the potential of utilizing the immune system for tumor suppression, albeit more so in the context of tumor vaccines than cellular immunotherapies. Furthermore, radiotherapy offers a wide range of options for treatment, each one chosen to complement the treatment regimen of choice for the individual patient. Lastly, new technologies applied in surgical reconstruction have a strong potential of improving the quality of life for OS patients following surgery, as technologies that are used in JPS can preserve affected joints and permit the retention of physiological function to a great 
extent. All in all, there is a great promise held in the future of osteosarcoma treatments which is expected to be realized in the near future.

\section{References}

1. American Cancer Society (2008) Cancer Facts \& Figures 2008.

2. Morrow J J, Khanna C (2015) Osteosarcoma Genetics and Epigenetics: Emerging Biology and Candidate Therapies. Critical Reviews in Oncogenesis 20(3-4): 173-197.

3. Kim M S, Lee S Y, Cho W H, Song W S, Koh J S, et al. (2009) Prognostic effects of doctor-associated diagnostic delays in osteosarcoma. Archives of Orthopaedic and Trauma Surgery 129(10): 1421-1425.

4. Ferguson J L, Turner S P (2018) Bone Cancer: Diagnosis and Treatment Principles. American Family Physician 98(4): 205-213.

5. Luetke A, Meyers P A, Lewis I, Juergens H (2014) Osteosarcoma treatment - where do we stand? A state of the art review. Cancer Treatment Reviews 40(4): 523-532.

6. Werner M (2016) Avoiding pitfalls in bone tumor diagnostics from the pathologist's point of view. 56(6): 536-542.

7. Van der Waal I (2020) Professional diagnostic delay in osteosarcomas of the jaws. Medicina Oral Patología Oral y Cirugia Bucal 25(6): e834-e837.

8. Joseph M Bestic, Daniel E Wessell, Francesca D Beaman, Carter CG J (2020) American College of Radiology American College of Radiology ACR Appropriateness Criteria ${ }^{\circledR}$ Primary Bone Tumors.

9. Pullan J E, Budh D P (2020) Primary Bone Cancer. In Stat Pearls Publishing.

10. Diessner B J, Weigel B J, Murugan P, Zhang L, Poynter J N, et al. (2020) Associations of Socioeconomic Status, Public vs Private Insurance, and Race/Ethnicity with Metastatic Sarcoma at Diagnosis. JAMA Network Open, 3(8).

11. Wang J, Liu S, Shi J, Li J, Wang S, et al. (2019) The Role of miRNA in the Diagnosis, Prognosis, and Treatment of Osteosarcoma. Cancer Biotherapy \& Radiopharmaceuticals 34(10): 605-613.

12. Gao S S, Wang Y J, Zhang G X, Zhang W T (2020) Potential diagnostic value of miRNAs in peripheral blood for osteosarcoma: A meta-analysis. Journal of Bone Oncology 23.

13. Huang X, Yang W, Zhang Z, Shao Z (2020) Dysregulated circRNAs serve as prognostic and diagnostic markers in osteosarcoma by sponging microRNA to regulate the downstream signaling pathway. Journal of Cellular Biochemistry 121(2): 1834-1841.

14. Jaffe $N$ (1972) Recent advances in the chemotherapy of metastatic osteogenic sarcoma. Cancer 30(6): 1627-1631.

15. Link MP, Goorin AM, Miser AW, Green AA, Pratt CB, et al. (1986) The Effect of Adjuvant Chemotherapy on Relapse-Free Survival in Patients with Osteosarcoma of the Extremity. New England Journal of Medicine 314(25): 1600-1606.

16. Ferrari S, Serra M (2015) An update on chemotherapy for osteosarcoma. Expert Opinion on Pharmacotherapy 16(18): 2727-2736.

17. Isakoff MS, Bielack SS, Meltzer P, Gorlick R (2015) Osteosarcoma: Current Treatment and a Collaborative Pathway to Success. Journal of Clinical Oncology 33(27): 3029-3035.

18. Gerrand C, Athanasou N, Brennan B, Grimer R, Judson I, et al. (2016) UK guidelines for the management of bone sarcomas. Clinical Sarcoma Research 6(1): 7.

19. Geller DS, Gorlick R (2010) Osteosarcoma: A Review of Diagnosis, Management, and Treatment Strategies. Clinical Oncol 8(10): 705-718.

20. Marina NM, Smeland S, Bielack SS, Bernstein M, Jovic G, et al. (2016) Comparison of MAPIE versus MAP in patients with a poor response to preoperative chemotherapy for newly diagnosed high-grade osteosarcoma (EURAMOS-1): An open-label, international, randomised controlled trial. The Lancet Oncology 17(10): 1396-1408.

21. Wei MY, Zhuang YF, Wang WM (2014) Gemcitabine for the treatment of patients with osteosarcoma. Asian Pacific Journal of Cancer Prevention: APJCP, 15(17): 7159-7162.

22. Durfee RA, Mohammed M, Luu HH (2016) Review of Osteosarcoma and Current Management. Rheumatology and Therapy 3(2): 221-243.

23. Wan X, Mendoza A, Khanna C, Helman LJ (2005) Rapamycin Inhibits Ezrin-Mediated Metastatic Behavior in a Murine Model of Osteosarcoma. Cancer Research 65(6): 2406-2411.

24. Fouladi M, Laningham F, Wu J, O Shaughnessy MA, Molina K, et al. (2007) Phase I Study of Everolimus in Pediatric Patients with Refractory Solid Tumors. Journal of Clinical Oncology 25(30): 4806-4812.

25. Houghton P J, Morton C L, Kolb E A, Gorlick R, Lock R, et al. (2008) Initial testing (stage 1) of the mTOR inhibitor rapamycin by the pediatric preclinical testing program. Pediatric Blood \& Cancer 50(4): 799-805.

26. Grignani G, Palmerini E, Dileo P, Asaftei S D, D Ambrosio L, et al. (2012) A phase II trial of sorafenib in relapsed and unresectable high-grade osteosarcoma after failure of standard multimodal therapy: An Italian Sarcoma Group study. Annals of Oncology 23(2): 508-516.

27. Safwat A, Boysen A, Lücke A, Rossen P (2014) Pazopanib in metastatic osteosarcoma: Significant clinical response in three consecutive patients. Acta Oncologica 53(10): 1451-1454.

28. Kansara M, Teng M W, Smyth M J, Thomas D M (2014) Translational biology of osteosarcoma. Nature Reviews Cancer 14(11): 722-735.

29. Caronia D, Patiño Garcia A, Peréz Martínez A, Pita G, Moreno LT, et al. (2011) Effect of ABCB1 and ABCC3 Polymorphisms on Osteosarcoma Survival after Chemotherapy: A Pharmacogenetic Study. PLOS ONE $6(10)$.

30. Kleinerman ES, Jia SF, Griffin J, Seibel NL, Benjamin RS, et al. (1992) Phase II study of liposomal muramyl tripeptide in osteosarcoma: The cytokine cascade and monocyte activation following administration. Journal of Clinical Oncology 10(8): 1310-1316.

31. Meyers PA, Schwartz CL, Krailo MD, Healey JH, Bernstein ML, et al. (2008) Osteosarcoma: The Addition of Muramyl Tripeptide to Chemotherapy Improves Overall Survival-A Report From the Children's Oncology Group. Journal of Clinical Oncology 26(4): 633-638.

32. Chou A J, Kleinerman E S, Krailo M D, Chen Z, Betcher DL, et al. (2009) Addition of muramyl tripeptide to chemotherapy for patients with newly diagnosed metastatic osteosarcoma. Cancer 115(22): 5339-5348.

33. Eilber F R, Townsend C, Morton D L (1975) Osteosarcoma. Results of treatment employing adjuvant immunotherapy. Clinical Orthopaedics and Related Research 111: 94-100.

34. Karbach J, Neumann A, Brand K, Wahle C, Siegel E, et al. (2012) Phase I Clinical Trial of Mixed Bacterial Vaccine (Coley's Toxins) in Patients with NY-ESO-1 Expressing Cancers: Immunological Effects and Clinical Activity. Clinical Cancer Research 18(19): 5449-5459.

35. Bielack S S, Smeland S, Whelan J S, Marina N, Jovic G, et al. (2015) Methotrexate, Doxorubicin, and Cisplatin (MAP) Plus Maintenance Pegylated Interferon Alfa-2b Versus MAP Alone in Patients with Resectable High-Grade Osteosarcoma and Good Histologic Response to Preoperative MAP: First Results of the EURAMOS-1 Good Response Randomized Controlled Trial. Journal of Clinical Oncology 33(20): 22792287.

36. Meazza C, Cefalo G, Massimino M, Daolio P, Pastorino U, et al. (2017) Primary metastatic osteosarcoma: Results of a prospective study in children given chemotherapy and interleukin-2. Medical Oncology 34(12): 191.

37. Robbins PF, Kassim SH, Tran T L N, Crystal J S, Morgan R A, et al. (2015) A Pilot Trial Using Lymphocytes Genetically Engineered with an NY-ESO- 
1-Reactive T-cell Receptor: Long-term Follow-up and Correlates with Response. Clinical Cancer Research 21(5): 1019-1027.

38. Ahmed N, Brawley VS, Hegde M, Robertson C, Ghazi A, et al. (2015) Human Epidermal Growth Factor Receptor 2 (HER2) -Specific Chimeric Antigen Receptor-Modified T Cells for the Immunotherapy of HER2Positive Sarcoma. Journal of Clinical Oncology 33(15): 1688-1696.

39. Miwa S, Shirai T, Yamamoto N, Hayashi K, Takeuchi A, et al. (2019) Current and Emerging Targets in Immunotherapy for Osteosarcoma. Journal of Oncology.

40. Dhupkar P, Gordon N, Stewart J, Kleinerman E S (2018) Anti-PD-1 therapy redirects macrophages from an M2 to an M1 phenotype inducing regression of OS lung metastases. Cancer Medicine 7(6): 2654-2664.

41. Lussier DM, Johnson J L, Hingorani P, Blattman J N (2015) Combination immunotherapy with $\alpha$-CTLA- 4 and $\alpha$-PD-L1 antibody blockade prevents immune escape and leads to complete control of metastatic osteosarcoma. Journal for Immuno Therapy of Cancer 3(1): 21.

42. Marulanda G A, Henderson E R, Johnson D A, Letson G D, Cheong D (2008) Orthopedic Surgery Options for the Treatment of Primary Osteosarcoma: Cancer Control 15(1): 13-20.

43. Tiwari A (2012) Current concepts in surgical treatment of osteosarcoma. Journal of Clinical Orthopaedics and Trauma 3(1): 4-9.

44. Li X, Zhang Y, Wan S, Li H, Li D, et al. (2016) A comparative study between limb-salvage and amputation for treating osteosarcoma. Journal of Bone Oncology 5(1): 15-21.

45. Simon M A, Aschliman M A, Thomas N, Mankin H J (1986) Limb-salvage treatment versus amputation for osteosarcoma of the distal end of the femur. JBJS 68(9): 1331-1337.

46. Lamplot J D, Denduluri S, Qin J, Li R, Liu X, et al. (2013) The Current and Future Therapies for Human Osteosarcoma. Current Cancer Therapy Reviews 9(1): 55-77.

47. Han G, Bi W Z, Xu M, Jia J P, Wang Y (2016) Amputation Versus LimbSalvage Surgery in Patients with Osteosarcoma: A Meta-analysis. World Journal of Surgery 40(8): 2016-2027.

48. Gaston C L, Nakamura T, Reddy K, Abudu A, Carter S, et al. (2014) Is limb salvage surgery safe for bone sarcomas identified after a previous surgical procedure? The Bone \& Joint Journal 96-B(5): 665-672.

49. He X, Gao Z, Xu H, Zhang Z, Fu P (2017) A meta-analysis of randomized control trials of surgical methods with osteosarcoma outcomes. Journal of Orthopaedic Surgery and Research 12(1): 5.

50. Misaghi A, Goldin A, Awad M, Kulidjian A A (2020) Osteosarcoma: A comprehensive review. SICOT-J 4.

51. Papakonstantinou E, Stamatopoulos A, I Athanasiadis D, Kenanidis E, Potoupnis M, et al. (2020). Limb-salvage surgery offers better fiveyear survival rate than amputation in patients with limb osteosarcoma treated with neoadjuvant chemotherapy. A systematic review and metaanalysis. Journal of Bone Oncology 25.

52. Kapoor S, Tiwari A, Kapoor S (2008) Primary tumours and tumorous lesions of clavicle. International Orthopaedics 32(6): 829-834.

53. Huang J, Bi W, Han G, Jia J, Xu M, et al. (2018) The multidisciplinary treatment of osteosarcoma of the proximal tibia: A retrospective study. BMC Musculoskeletal Disorders 19(1): 315.

54. Takeuchi A, Yamamoto N, Hayashi K, Matsubara H, Miwa S, et al. (2019) Joint-preservation surgery for pediatric osteosarcoma of the knee joint. Cancer and Metastasis Reviews 38(4): 709-722.

55. Higuchi T, Yamamoto N, Nishida H, Hayashi K, Takeuchi A, et al. (2017) Knee joint preservation surgery in osteosarcoma using tumor-bearing bone treated with liquid nitrogen. International Orthopaedics 41(10): 2189-2197.

56. Takeuchi A, Yamamoto N, Hayashi K, Matsubara H, Kimura H, et al. (2018) Growth of epiphysis after epiphyseal-preservation surgery for childhood osteosarcoma around the knee joint. BMC Musculoskeletal Disorders 19(1): 185.

57. Kamal A F, Rubiansyah P (2019) Clinical outcome of various limb salvage surgeries in osteosarcoma around knee: Megaprosthesis, extracorporeal irradiation and resection arthrodesis. Annals of Medicine and Surgery 42: 14-18.

58. Xin S, Wei G (2020) Prognostic factors in osteosarcoma: A study level meta-analysis and systematic review of current practice. Journal of Bone Oncology 21.

59. Oertel S, Blattmann C, Rieken S, Jensen A, Combs S E, et al. (2010) Radiotherapy in the treatment of primary osteosarcoma-A single center experience. Tumori 96(4): 582-588.

60. Ciernik I F, Niemierko A, Harmon D C, Kobayashi W, Chen Y L, et al. (2011) Proton-Based Radiotherapy for Unresectable or Incompletely Resected Osteosarcoma. Cancer 117(19): 4522-4530.

61. Mc Govern S L, Mahajan A (2012) Progress in Radiotherapy for Pediatric Sarcomas. Current Oncology Reports 14(4): 320-326.

62. Tinkle C L, Lu J, Han Y, Li Y, Mc Carville B M, et al. (2019) Curative-intent radiotherapy for pediatric osteosarcoma: The St. Jude experience. Pediatric Blood \& Cancer 66(8).

63. Schwarz R, Bruland O, Cassoni A, Schomberg P, Bielack S (2010) The Role of Radiotherapy in Oseosarcoma. Pediatric and Adolescent Osteosarcoma.

64. Posthuma De Boer J, Würdinger T, Graat HC, Van Beusechem V W, Helder M N, et al. (2011) WEE1 inhibition sensitizes osteosarcoma to radiotherapy. BMC Cancer 11: 156.

65. Whelan J S, Davis L E (2018) Osteosarcoma, Chondrosarcoma, and Chordoma. Journal of Clinical Oncology 36(2): 188-193.
ISSN: 2574-1241

DOI: $10.26717 /$ BJSTR.2020.32.005268

Konstantinos Kapetanos. Biomed J Sci \& Tech Res

This work is licensed under Creative Commons Attribution 4.0 License

Submission Link: https://biomedres.us/submit-manuscript.php

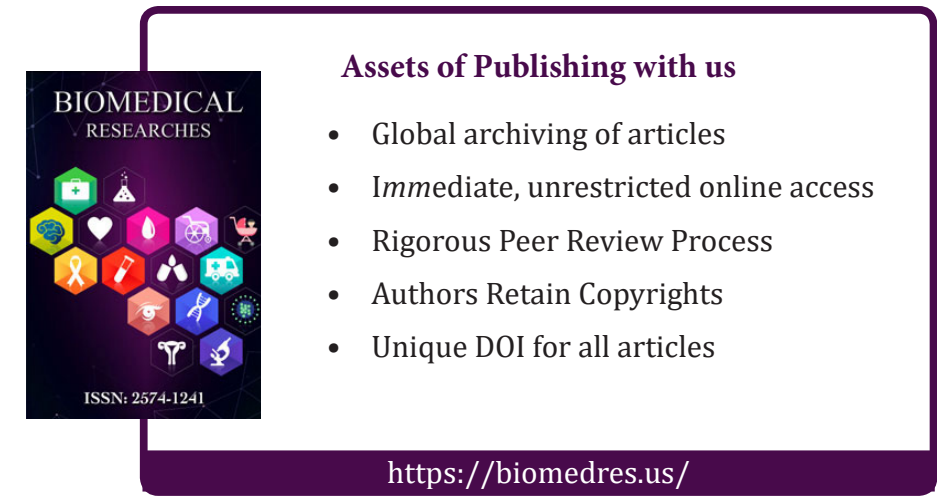

